\title{
Analysis of Inductively Coupled RFID Marker Localization Methods
}

\author{
Peter Vestenický \\ University of Žilina, Faculty of \\ Electrical Engineering, Department \\ of Control and Information \\ Systems, Univerzitná 8215/1, \\ 01026 Žilina, Slovakia \\ Email: \\ peter.vestenicky@fel.uniza.sk
}

\author{
Tomáš Mravec \\ University of Žilina, Faculty of \\ Electrical Engineering, Department \\ of Control and Information \\ Systems, Univerzitná 8215/1, \\ 01026 Žilina, Slovakia \\ Email: \\ tomas.mravec@fel.uniza.sk
}

\author{
Martin Vestenický \\ University of Žilina, Faculty of \\ Electrical Engineering, Department \\ of Telecommunications and \\ Multimedia, Univerzitná 8215/1, \\ 01026 Žilina, Slovakia \\ Email: \\ martin.vestenicky@fel.uniza.sk
}

\begin{abstract}
The presented paper is focused on analysis of two methods of marker localization. The markers are passive RFID transponders (without or with identification chip) consisting of tuned LC circuit and being used to mark and trace underground networks such as cables and pipes. Localization of the marker is based on evaluation of signal amplitude received from the excited marker, i. e. it is RSSI based localization method. The excitation of marker can be periodically repeated or continuous. In the first case the localization process consists of two stages - excitation and receiving of marker damped oscillations, in the second case the amplitude of continuously generated excitation signal is decreased by vicinity of the marker. Both localization methods are mathematically analyzed by modeling of their circuits using differential equations. The results of analysis are used to compare both methods and to evaluate their suitability for practical utilization.
\end{abstract}

\section{INTRODUCTION}

I NDUCTIVELY coupled RFID (Radio Frequency Identification) systems [1] are now being widely used in many industrial applications. For example, the marking of goods by RFID technology enables the traceability of goods which is helpful to control the whole logistic chain from production to sale. In addition to these applications, the RFID transponders are being used for marking of underground facilities location. Such RFID transponders are called "markers". The marker is a passive RFID transponder consisting of a tuned LC circuit without identification chip (1-bit) or with identification chip tuned on low frequency in $77 \mathrm{kHz}-170 \mathrm{kHz}$ band.

For localization of some older underground facilities (cables, pipes etc.) a signal can be injected into their continual metal conductor and this signal can then be received on terrain surface and the cable or pipe can be traced. Today's underground facilities are mostly constructed from plastic material so this simple localization and tracing method cannot be used, therefore in this case the marking of underground objects by RFID markers is the only useable method.

The unknown position of marker under the terrain surface can be estimated by a localization device (locator). Moreover, the depth of marker can be estimated by RSSI
(Received Signal Strength Indication) similarly as described in [2], [3]. In [4] the authors describe marker localization methods based on marker damped oscillations and on continuous generating of electromagnetic waves. This work extends the analyses of RSSI based marker localization methods. The method based on damped oscillations of marker is extended by introduction of separate damping and sensing resistors and the analysis of method based on continuous marker excitation is done by applying differential equations instead of algebraic equations to analyze the transient phenomena in sensing circuit.

\section{RELATED WORKS}

The localization of moving underground objects (for example animals) based on inductive coupling is described in [5]. This application assumes the use of sensor network consisting of transmitting coils fixed on terrain surface and the moving underground object equipped with receiver collects data transmitted from these coils.

Indoor localization based on triaxial coils applied in both transmitter and receiver with very low working frequency 2.5 $\mathrm{kHz}$ is published in [6]. Low frequency magnetic field is suitable for underground localization purposes, too, because it is not affected by ground properties.

Another approach is presented in [7]. This work assumes localization based on UHF RFID tags in mining industry, but it is performed in mining tunnels and localization from the terrain surface is not assumed so the UHF signals do not propagate through layer of ground.

\section{MUtual InductanCE}

An important quantity in the models presented in next chapters is the mutual inductance $M$ between marker and locator coils which depends on the geometrical arrangement of coils according to equation [1], [4], [8]:

$$
M=\frac{\sqrt{L_{R} L_{T}} r_{R}^{2} r_{T}^{2} \cos \theta}{\sqrt{r_{R} r_{T}}\left(r_{R}^{2}+x^{2}\right)^{\frac{3}{2}}}
$$


where $r_{R}$ is radius of RFID locator antenna coil, $r_{T}$ is radius of marker coil, $x$ is distance between the locator antenna and the marker and $\theta$ is the angle between coil of locator antenna and marker coil (note that if $\theta=0{ }^{\circ}$ then coils are parallel).

\section{MATHEMATICAl Model BASED ON DAMPED OSCILLATION}

This principle of localization assumes that the localization device periodically excites the marker LC circuit and in the pauses between excitation periods the response from marker in form of its damped oscillations is received. The simple model with one resistor was analyzed in [4]. In this chapter a more complex model with separate damping and sensing resistors will be analyzed. The first resistor $R_{D}$ is used for fast damping of locator $L_{R} C_{R}$ circuit damped oscillations and its value was calculated from equation (2). The second resistor $R_{M}$ is used for current sensing in the receiving stage of localization. The model is shown in Fig. 1.

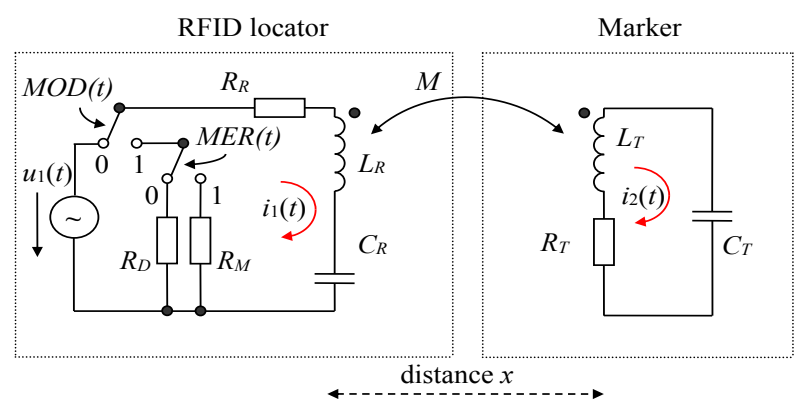

Fig. 1 Model of localization with separate damping and measuring resistors

$$
R_{D}=2 \sqrt{\frac{L_{R}}{C_{R}}}-R_{R}
$$

For this model the next system of equations (3) can be derived

$$
\begin{gathered}
L_{R} \frac{d i_{1}(t)}{d t}+\left[(1-M E R(t)) M O D(t) R_{D}+M E R(t) M O D(t) R_{D}+R_{R}\right] i_{1}(t)+ \\
+\frac{1}{C_{R}} \int_{0}^{t} i_{1}(\tau) d \tau-M \frac{d i_{2}(t)}{d t}=(1-M O D(t)) u_{1}(t) \\
L_{T} \frac{d i_{2}(t)}{d t}+R_{T} i_{2}(t)+\frac{1}{C_{T}} \int_{0}^{t} i_{2}(\tau) d \tau-M \frac{d i_{1}(t)}{d t}=0
\end{gathered}
$$

The modulation function $M O D(t)$ is given by equation (4) and the sensing (measuring) resistor $R_{M}$ is switched by function $\operatorname{MER}(t)$ given by the equation (5)

$$
M O D(t)=\frac{\operatorname{Sign}\left(-\sin \frac{2 \pi f t}{250}\right)+1}{2}
$$

$$
\operatorname{MER}(t)=\frac{\operatorname{Sign}\left(-\sin \frac{2 \pi f(t-\Delta T)}{250}\right)+1}{2}
$$

i. e. it is binary square signal with a frequency 250 times lower than the frequency of excitation signal source and shifted (delayed) by $\Delta T$ in time against the modulation function $M O D(t)$. During the small delay $\Delta T$ the oscillations of locator tuned circuit has to be damped.

The excitation signal source is assumed harmonic, i. e.

$$
u_{1}(t)=U_{1} \sin (2 \pi f t)
$$

The system of integrodifferential equations (3) was numerically solved after its conversion to the $1^{\text {st }}$ order system of differential equations (7) by substitution $x_{1}(t)=i_{1}(t)$, $x_{2}(t)=\mathrm{d} i_{1}(t) / \mathrm{d} t, x_{3}(t)=i_{2}(t), x_{4}(t)=\mathrm{d} i_{2}(t) / \mathrm{d} t, \omega=2 \pi f$. Then we get:

$$
\begin{aligned}
\frac{d x_{1}(t)}{d t}= & x_{2}(t) \\
\frac{d x_{2}(t)}{d t}= & -a_{1} x_{1}(t)+a_{2}(1-M O D(t)) \omega U_{1} \cos (\omega t)-a_{3} x_{3}(t)-a_{4} x_{4}(t)- \\
& -a_{2}\left[R_{R}+[1-M E R(t)] M O D(t) R_{D}+M E R(t) M O D(t) R_{M}\right] x_{2}(t) \\
\frac{d x_{3}(t)}{d t}= & x_{4}(t) \\
\frac{d x_{4}(t)}{d t}= & -b_{1} x_{1}(t)+b_{2}(1-M O D(t)) \omega U_{1} \cos (\omega t)-b_{3} x_{3}(t)-b_{4} x_{4}(t)- \\
& -b_{2}\left[R_{R}+[1-M E R(t)] M O D(t) R_{D}+M E R(t) M O D(t) R_{M}\right] x_{2}(t)
\end{aligned}
$$

where the individual coefficients $a_{1}, a_{2}, a_{3}, a_{4}$ and $b_{1}, b_{2}$, $b_{3}, b_{4}$ are given by (8).

$$
\begin{array}{ll}
a_{1}=\frac{L_{T}}{C_{R}\left(L_{R} L_{T}-M^{2}\right)} & b_{1}=\frac{M}{C_{R}\left(L_{R} L_{T}-M^{2}\right)} \\
a_{2}=\frac{L_{T}}{L_{R} L_{T}-M^{2}} & b_{2}=\frac{M}{L_{R} L_{T}-M^{2}} \\
a_{3}=\frac{M}{C_{T}\left(L_{R} L_{T}-M^{2}\right)} & b_{3}=\frac{L_{R}}{C_{T}\left(L_{R} L_{T}-M^{2}\right)} \\
a_{4}=\frac{M R_{T}}{L_{R} L_{T}-M^{2}} & b_{4}=\frac{L_{R} R_{T}}{L_{R} L_{T}-M^{2}}
\end{array}
$$

The used values of $R_{R}, L_{R}, C_{R}$ and $R_{T}, L_{T}, C_{T}$ are listed in the next table:

TABLE I.

VALUES OF COMPONENTS USED IN NUMERICAL CALCULATIONS

\begin{tabular}{|c|c|c|c|c|c|}
\hline $\boldsymbol{R}_{\boldsymbol{R}}$ & $\boldsymbol{L}_{\boldsymbol{R}}$ & $\boldsymbol{C}_{\boldsymbol{R}}$ & $\boldsymbol{R} \boldsymbol{T}$ & $\boldsymbol{L}_{\boldsymbol{T}}$ & $\boldsymbol{C}_{\boldsymbol{T}}$ \\
\hline $15.7 \Omega$ & $1 \mathrm{mH}$ & $1.621 \mathrm{nF}$ & $7.85 \Omega$ & $1 \mathrm{mH}$ & $1.621 \mathrm{nF}$ \\
\hline
\end{tabular}

Note that the values of $L_{R}, C_{R}$ and $L_{T}, C_{T}$ were selected so that the corresponding resonant frequencies are $f_{R}=f_{T}=125 \mathrm{kHz}$.

The radiuses of both coils used in numerical calculations are $r_{R}=r_{T}=0.1 \mathrm{~m}$, distance between them is $x=0.4 \mathrm{~m}$ and the angle $\theta=0^{\circ}$. Corresponding mutual inductance $\mathrm{M}$ is then calculated from (1). Amplitude of the excitation signal is $U_{1}=10 \mathrm{~V}$, its frequency $f$ is $125 \mathrm{kHz}$.

The damping resistor $R_{D}=1533 \Omega$ calculated from (2) in this case ensures the minimum time of $L_{R} C_{R}$ transient 
response. The used values of the measuring resistor are $R_{M}=100 \Omega$ for comparison with the results obtained in [4] and $R_{M}=1 \Omega$ to maximize the current response $i_{1}(t)$ from marker.

The time course of the current $i_{1}(t)$ for model of marker localization with two resistors is shown in Fig. 2 and Fig. 3 for $R_{D}=1533 \Omega, R_{M}=100 \Omega$ and $R_{M}=1 \Omega$, respectively.

Moreover, the dependence of maximum current amplitude $I_{1 \_ \text {Max }}$ on the distance $x$ was calculated for this model and it is shown in Fig. 4. Note that the current maximum was calculated in the time interval after the transients of the excitation current decay. For comparison of the presented analysis results and the results from simplified model calculated in [4] the case when $R_{D}=R_{M}$ was calculated, too. This case is identical with the simplified model and the comparison is shown in Fig. 4.

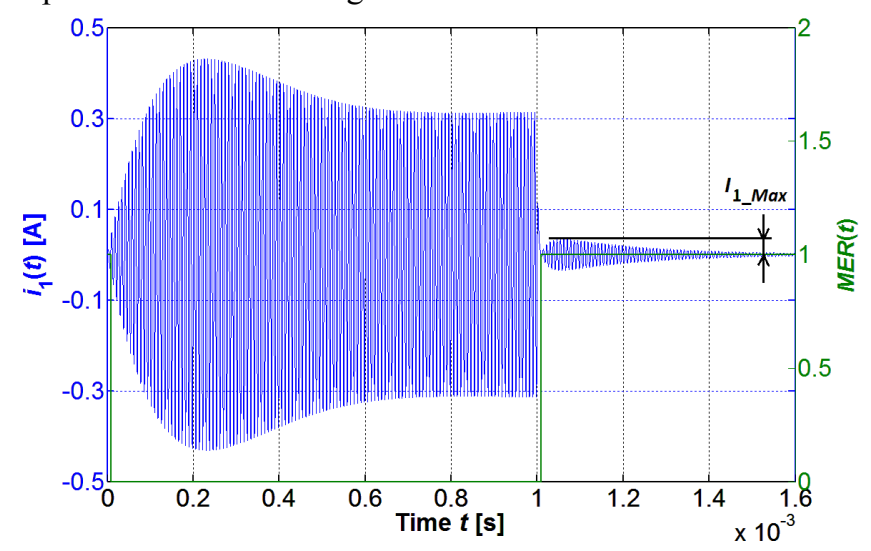

Fig. 2 Time course of the current $i_{1}(t)$ for $R_{D}=1533 \Omega$ and $R_{M}=100 \Omega$

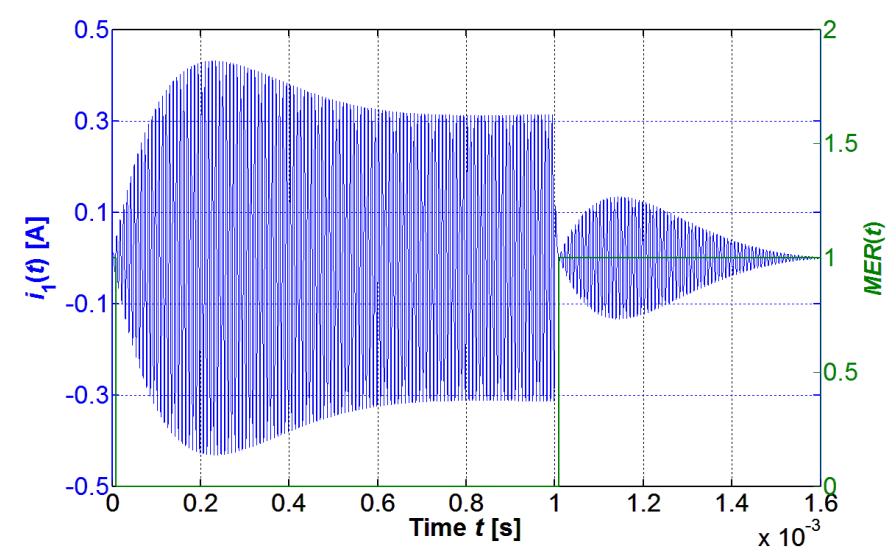

Fig. 3 Time course of the current $i_{1}(t)$ for $R_{D}=1533 \Omega$ and $R_{M}=1 \Omega$

The measuring resistor $R_{M}$ influences the maximum current amplitude $I_{1 \_ \text {Max }}$ because this resistors acts as additional damping resistor for $L_{R} C_{R}$ circuit so that the ideal situation is when $R_{M} \rightarrow 0$.

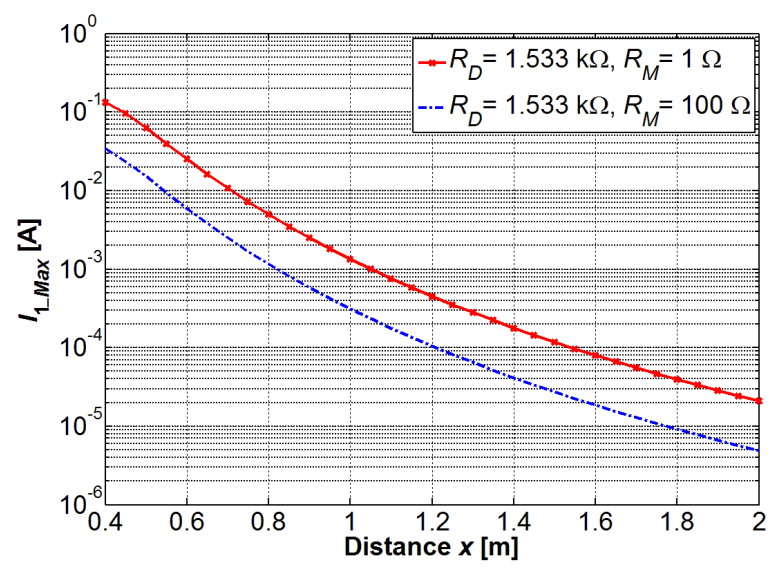

Fig. 4 Maximum amplitude of current response from marker with nominal resonant frequency $f_{T}=125 \mathrm{kHz}$ for two resistor model

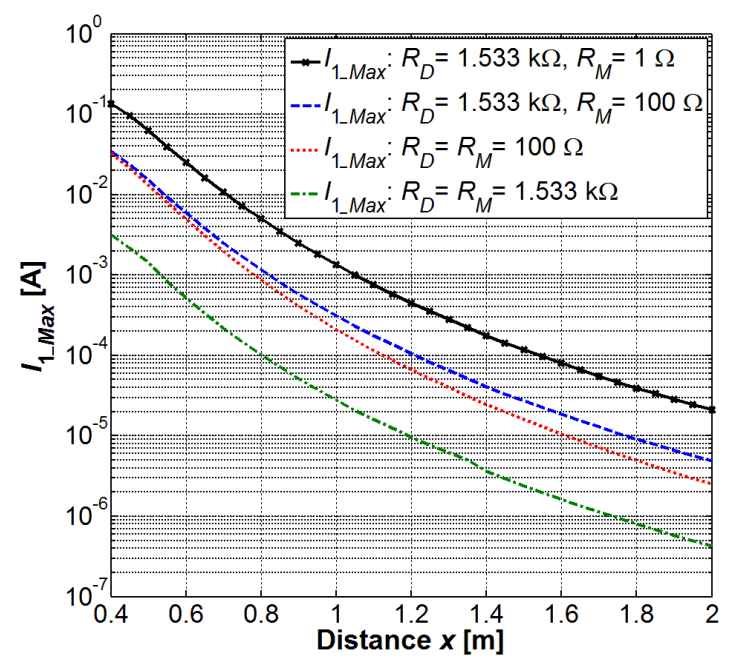

Fig. 5 Maximum amplitude of current response from marker for damped oscillation model

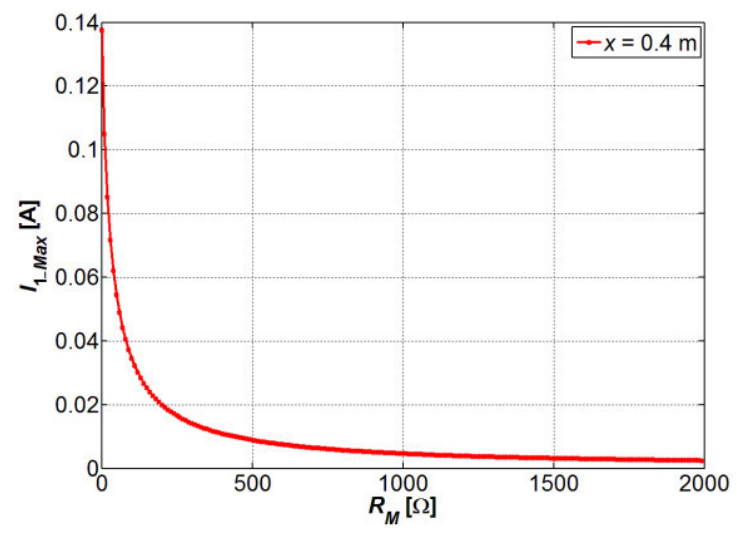

Fig. 6 Dependence of maximum current amplitude on the measuring resistor $R_{M}$ for distance $x=0.4 \mathrm{~m}$

\section{Mathematical Model Based On Continuous EXCITATION OF MARKER}

This localization principle is used when the RFID locator continuously generates the magnetic field by its antenna coil $L_{R}$. The amplitude of current in locator circuit is then 
influenced by resonant circuit of marker so that the steady state current amplitude in locator circuit decreases if the marker is nearby.

The model is derived from the previous case by eliminating the switches. Schematic diagram of this model is shown in Fig. 7. In dependence on the distance $x$ between coils the marker resonant circuit influences the amplitude of current $i_{1}(t)$ in locator antenna circuit.

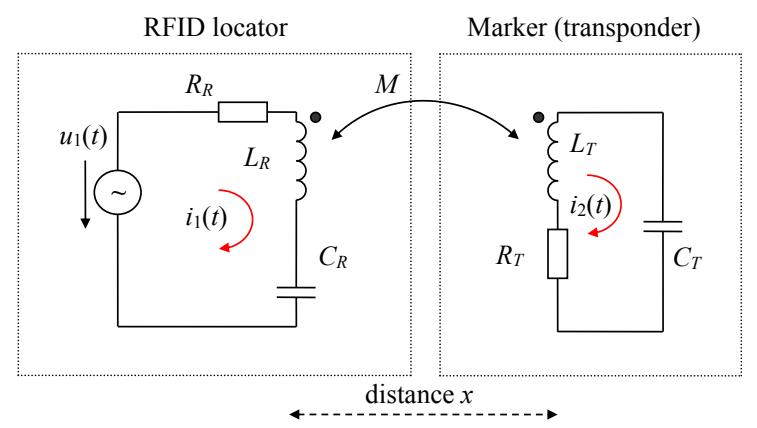

Fig. 7 Model of localization based on continuous excitation

This model can be analyzed by using of complex impedances of its components as it was performed in [4]. But such analysis did not show the transient phenomena in locator circuit and the results give only steady state solution. Therefore new model based on differential equations was created.

This model can be described by the following system of differential equations:

$$
\begin{aligned}
& L_{R} \frac{d i_{1}(t)}{d t}+R_{R} i_{1}(t)+\frac{1}{C_{R}} \int_{0}^{t} i_{1}(\tau) d \tau-M \frac{d i_{2}(t)}{d t}=u_{1}(\mathrm{t}) \\
& L_{T} \frac{d i_{2}(t)}{d t}+R_{T} i_{2}(t)+\frac{1}{C_{T}} \int_{0}^{t} i_{2}(\tau) d \tau-M \frac{d i_{1}(t)}{d t}=0
\end{aligned}
$$

The signal source in Fig. 7 is harmonic as defined by (6). Similar as in previous chapter the system of integrodifferential equations (9) was transformed by substitution $\quad x_{1}(t)=i_{1}(t), \quad x_{2}(t)=d i_{1}(t) / d t, \quad x_{3}(t)=i_{2}(t)$, $x_{4}(t)=d i_{2}(t) / \mathrm{d} t, \omega=2 \pi f$. Then we get the $1^{\text {st }}$ order system of differential equations (10) which can be easily solved by standard mathematic software.

$$
\begin{aligned}
& \frac{d x_{1}(t)}{d t}=x_{2}(t) \\
& \frac{d x_{2}(t)}{d t}=a_{1} x_{1}(t)+a_{2} x_{2}(t)+a_{3} x_{3}(t)+a_{4} x_{4}(t)-a_{5} \omega U_{1} \cos (\omega t) \\
& \frac{d x_{3}(t)}{d t}=x_{4}(t) \\
& \frac{d x_{4}(t)}{d t}=b_{1} x_{1}(t)+b_{2} x_{2}(t)+b_{3} x_{3}(t)+b_{4} x_{4}(t)-b_{5} \omega U_{1} \cos (\omega t)
\end{aligned}
$$

where individual coefficients are given by (11). Note that these coefficients are different from the coefficients given by (8).

$$
\begin{array}{ll}
a_{1}=\frac{L_{T}}{C_{R}\left(M^{2}-L_{R} L_{T}\right)} & b_{1}=\frac{M}{C_{R}\left(M^{2}-L_{R} L_{T}\right)} \\
a_{2}=\frac{L_{T} R_{R}}{M^{2}-L_{R} L_{T}} & b_{2}=\frac{R_{R} M}{M^{2}-L_{R} L_{T}} \\
a_{3}=\frac{M}{C_{T}\left(M^{2}-L_{R} L_{T}\right)} & b_{3}=\frac{L_{R}}{C_{T}\left(M^{2}-L_{R} L_{T}\right)} \\
a_{4}=\frac{M R_{T}}{M^{2}-L_{R} L_{T}} & b_{4}=\frac{L_{R} R_{T}}{M^{2}-L_{R} L_{T}} \\
a_{5}=\frac{L_{T}}{M^{2}-L_{R} L_{T}} & b_{5}=\frac{M}{M^{2}-L_{R} L_{T}}
\end{array}
$$

In this case the detection of marker vicinity is more complicated as in the previous chapter because there is not the time stage in which only the response from excited marker can be received but the excitation signal in locator circuit is always present. The symptom of marker vicinity is decreasing of steady state current amplitude in locator circuit. When the distance between marker and locator is big $(x \rightarrow \infty)$ then the mutual inductance calculated from (1) is very small $(M \rightarrow 0)$. In this case the current $i_{1}(t)$ has maximum value $I_{1 \max }$, which is measured in steady state after the time $t=2.5 \mathrm{~ms}$. The marker vicinity then causes current drop $\Delta I_{1}(12)$ which can be calculated as difference between steady value of current $i_{1}(t)$ (see Fig. 8) and its maximum $I_{1 \max }$ measured in the same time point when no marker is nearby the locator.

$$
\Delta I_{1}=I_{1 \max }-I_{1}
$$

The time course of current $i_{1}(t)$ in the RFID locator circuit (Fig. 8) was numerically calculated from the system (10) for the same parameters as used in previous chapter. The dependence of current drop $\Delta I_{1}$ versus distance $x$ is shown in Fig. 9.

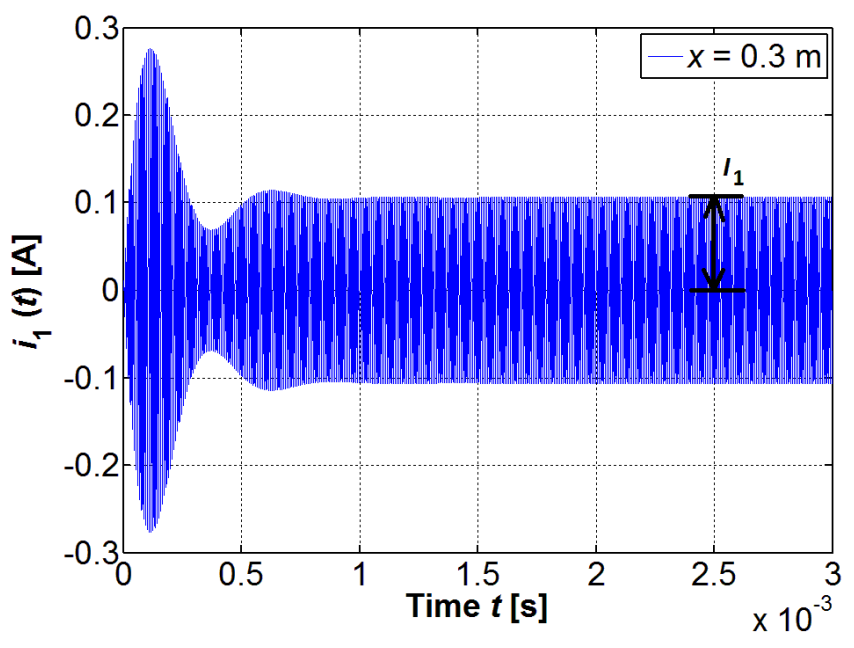

Fig. 8 The time course of current $i_{1}(\mathrm{t})$, distance $x=0.3 \mathrm{~m}$ 


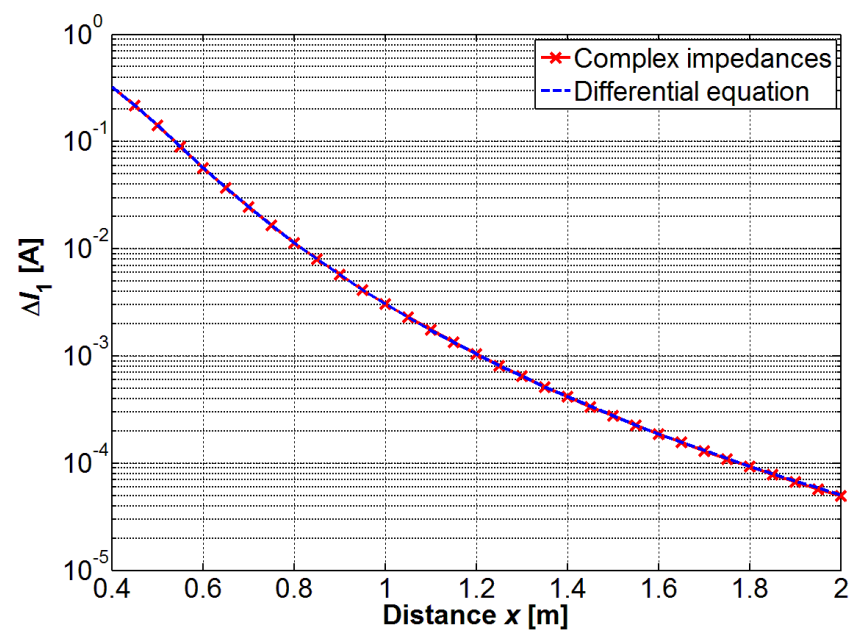

Fig. 9 Current drop $\Delta I_{1}$ versus distance $x$

The results calculated by analysis based on differential equations (10) were compared with the results obtained by analysis based on algebraic equations and complex impedances from [4]. This data is shown in the same graph in Fig. 9. As shown in this figure, both analyses give the same results.

\section{CONCLUSION}

Presented paper extends the mathematical analyses of marker localization principles. Two possible marker localization methods were analysed. For practical use the first method seems to be appropriate because the signal in receiving stage of localization can be directly processed.

The second method based on continuous marker excitation would require more complicated signal processing in locator. This complication is caused by "mixing" the excitation and response signals so that the marker vicinity cannot be simply detected by signal presence detection as in the first case but by detection of signal drop. Moreover the transient phenomena occur as documented in Fig. 8.

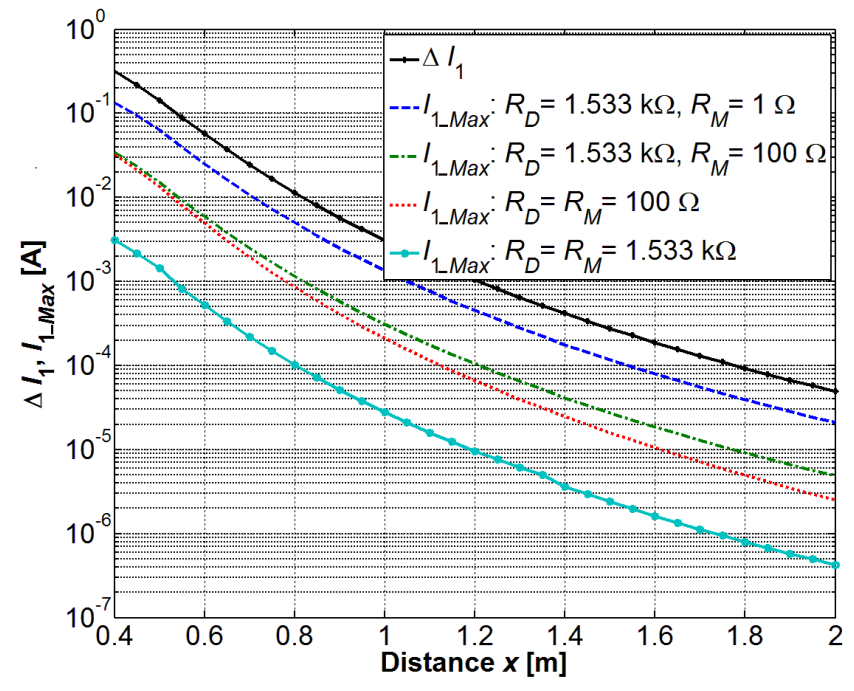

Fig. 10 Comparison of both localization models
Another criterion for evaluation of presented localization methods is based on their sensitivity to the marker vicinity. From comparison in Fig. 10 the second method seems to be more sensitive to the marker vicinity. Because the markers are typical near field application, the current amplitude in both cases decreases very rapidly when the distance between marker and locator increases.

The next research will be focused on the design of hardware needed to perform series of measurements so that the results of performed analyses will be compared with real measured data.

\section{ACKNOWLEDGMENT}

This work has been supported by the Cultural and Educational Grant Agency of the Slovak Republic (KEGA) Number: 008ŽU-4/2015: "Innovation of $H W$ and SW tools and methods of laboratory education focused on safety aspects of ICT within safety critical applications of processes control'.

\section{REFERENCES}

[1] K. Finkenzeller, RFID Handbook Fundamentals and Applications in Contactless Smart Cards, Radio Frequency Identification and NearField Communication, Third Edition. John Wiley and Sons, Ltd. Chichester, UK, 2010, ISBN 978-0-470-69506-7. http://dx.doi.org/10.1002/9780470665121

[2] M. Y. Ahmad, A. S. Mohan, "RFID Reader Localization Using Passive RFID Tags". In: APMC 2009, Asia Pacific Microwave Conference, Singapore, pp. $606-609$, December $7^{\text {th }}-10^{\text {th }} 2009$, ISBN 978-1-4244-2802-1. http://dx.doi.org/10.1109/APMC.2009.5384152

[3] T. Van Haute, J. Rossey, P. Becue, E. De Poorter, I. Moerman, P. Demeester, "A Hybrid Indoor Localization Solution Using a Generic Architectural Framework for Sparse Distributed Wireless Sensor Networks". In: FedCSIS 2014, Federated Conference on Computer Science and Information Systems, September 7th - 10th, 2014, Warsaw, Poland, pp. 1009 - 1015, ISBN 978-83-60810-58-3. http://dx.doi.org/10.15439/2014F20

[4] P. Vestenický, T. Mravec, M. Vestenický, "Mathematical Modelling of Single-bit Passive RFID Marker Localization Methods". In: ELEKTRO 2014, $10^{\text {th }}$ International Conference, May $19^{\text {th }}-20^{\text {th }}$, 2014, Rajecké Teplice, Slovakia, pp. 504 - 507, ISBN 978-1-47993720-2. http://dx.doi.org/10.1109/ELEKTRO.2014.6848946

[5] A. Markham, N. Trigoni, D. W. Macdonald, S. A. Ellwood, "Underground Localization in 3-D Using Magneto-Inductive Tracking”. IEEE Sensors Journal, vol. 12, no. 6, pp. 1809 - 1816, June 2012, ISSN 1530-437X.

http://dx.doi.org/10.1109/JSEN.2011.2178064

[6] T. E. Abrudan, A. Markham, N. Trigoni, "Poster Abstract: A Case for Magneto-Inductive Indoor Localization". In: EWSN 2014, The $11^{\text {th }}$ European Conference on Wireless Sensor Networks, University of Oxford, Oxford, UK, pp. $18-19$, February $17^{\text {th }}-19^{\text {th }}, 2014$, ISBN 978-3-319-04651-8

[7] J. Hautcoeur, L. Talbi, M. Nedil, "High Gain RFID Tag Antenna for the Underground Localization Applications at $915 \mathrm{MHz}$ Band". In: APSURSI 2013, IEEE Antennas and Propagation Society International Symposium, Orlando, FL, pp. 1488 - 1489, July $7^{\text {th }}-$ $13^{\text {th }}, 2013$, ISBN 978-1-4673-5315-1. http://dx.doi.org/10.1109/APS.2013.6711403

[8] RFID made easy. Application note AN411, EM MicroelectronicMarin SA. Marin, Switzerland, 2002. [online] URL http://www.emmicroelectronic.com/webfiles/product/rfid/an/an411.pd f 